\title{
Small-Sized Radio Telescopes for Monitoring and Studies of Solar Radio Emission at Meter and Decameter Wavelengths
}

\author{
S. Yerin ${ }^{1,2}$, A. Stanislavsky ${ }^{1,2}$, I. Bubnov ${ }^{1}$, A. Konovalenko ${ }^{1}$, \\ P. Tokarsky ${ }^{1}$, V. Zakharenko ${ }^{1}$ \\ ${ }^{1}$ Institute of Radio Astronomy of the NASU, Ukraine; \\ ${ }^{2}$ V.N. Karazin Kharkiv National University, Ukraine
}

\author{
E mail (yerin.serge@gmail.com).
}

Accepted: 10 January 2019

\begin{abstract}
The paper shows development prospects of solar studies with small-sized sensitive radio telescopes such as the GURT active antenna which is an element of the phased array of the GURT radio telescope. Starting with GURT active antenna sensitivity calculations for solar observations, we compare our solar radio emission observations with the records of other radio telescopes. We discuss the potential of using this single antenna as an instrument for studying the active Sun manifestations from the lunar surface and suggest its possible adjustment to observe poor studied solar bursts under the terrestrial ionosphere cutoff.
\end{abstract}

\section{(c) 2019 BBSCS RN SWS. All rights reserved}

Keywords:

\section{Introduction}

Low-frequency radio astronomy deals with many sporadic cosmic signals (Konovalenko et al., 2016). One of the strongest cosmic signal types received on Earth is the solar sporadic radio emission. The emission at low frequencies is generated at 3-5 solar radii height and helps to examine properties of the upper solar corona. Solar radio emission is classified into many types which still have not been studied and interpreted thoroughly. Some types of the emission were explained by several different models and yet none of them fully explains all features of these emissions spectra.

Over all the history of radio astronomy the scientists tried to build larger telescopes to achieve higher sensitivity and spatial selectivity of their instruments. This process has led to construction of large instruments and considering experimental radio astronomy as a highly cost-consuming science. At the same time, the flux of solar radio emission at frequencies below 100 $\mathrm{MHz}$ as well as their sporadic nature and specific features in timefrequency domain allow one to distinguish it from other emission sources, register and study with sensitive wideband small-sized antennas. Unfortunately, in this case, we do not have a good spatial resolution of antenna, but the dynamic spectra obtained in this way still carry much "footprints" due to physical processes taking place in the solar corona. In addition, the almost omnidirectional antenna is easier to operate and allows observing solar emission from dusk to dawn (Tokarsky et al., 2015).

\section{Antenna Design and Characteristics}

A small antenna, matched to a receiving system in terms of its parameters, can be sufficiently sensitive for recording sporadic manifestations of solar emission on the Earth. In case of smallsized antenna with poor spatial selectivity the RFI signals from all directions, especially the ones near horizon, become an urgent problem. That is why heightened dynamic range of the whole system is an obligatory requirement.

Modern low-frequency radio telescopes use active antenna arrays as sensors of cosmic radio emission (Konovalenko et al., 2016). Each element of the array is an active antenna that should satisfy all the requirements for small-sized solar radio telescope listed above. Giant Ukrainian Radio Telescope (GURT) is a project of new-generation low-frequency radio telescope developed in Ukraine by Institute of Radio Astronomy of National Academy of Sciences of Ukraine (IRA NASU) based on the

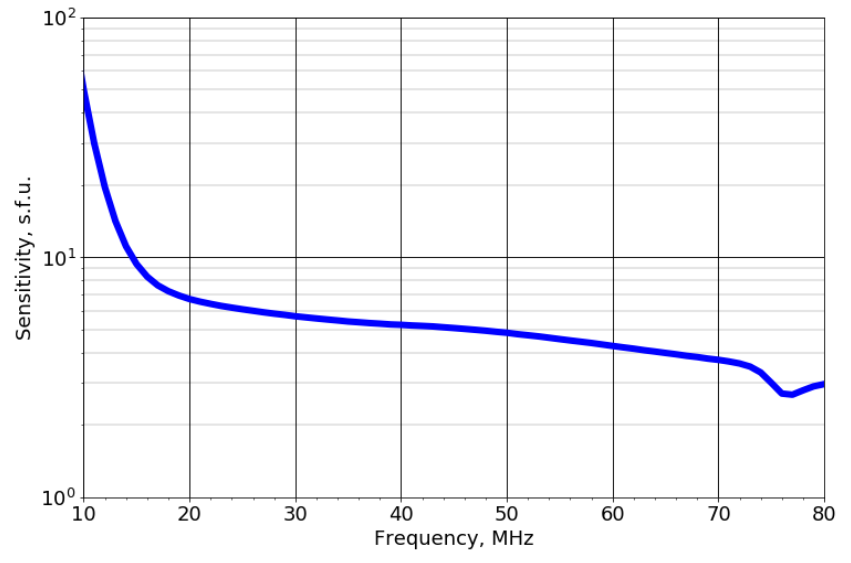

Figure 1: Sensitivity of the GURT active antenna for integration intervals in time and frequency of $100 \mathrm{~ms}$ and $4.9 \mathrm{kHz}$, respectively, as it follows from calculations in the paper (Tokarsky et al., 2017).

experience of UTR-2 radio telescope maintenance and upgrading (Ryabov et al., 2010; Konovalenko et al., 2016). GURT operates in $8-80 \mathrm{MHz}$ range and consists of separate subarrays of 25 active antenna elements. Each subarray has discrete phase shifting based on true time-delay lines switching principle which allows beam pointing to 213 directions on celestial hemisphere. A two-channel Advanced Digital Receiver (ADR) receiver was specially developed for receiving signals from each subarray to provide means of real-time digitization of the signal and waveform recording or on-the-fly spectra calculation and storing (Zakharenko et al., 2016). The GURT active antenna element is developed to ensure high sensitivity in the whole operation range, and its characteristics are well studied by means of EM modeling and measurements. On the basis of these studies the sensitivity of single antenna element was calculated for ordinary observation parameters (100 ms integration time and $9.7 \mathrm{kHz}$ frequency resolution, typical for solar observations with the ADR receiver). The results of calculations are shown in Fig. 1 and demonstrate that the sensitivity of the GURT active antenna is sufficient for registration of solar emission in the wide range of flux densities. 


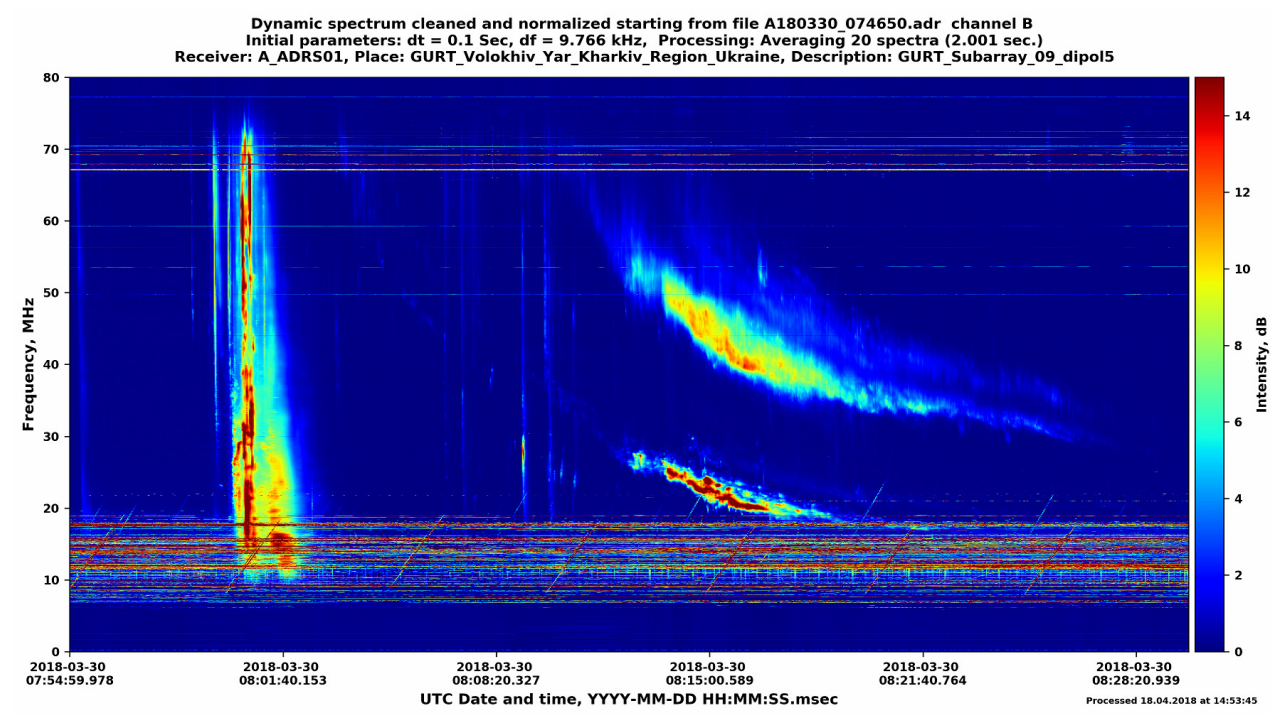

Dynamic spectrum cleaned and normalized starting from file B170712 082511 .adr channel A

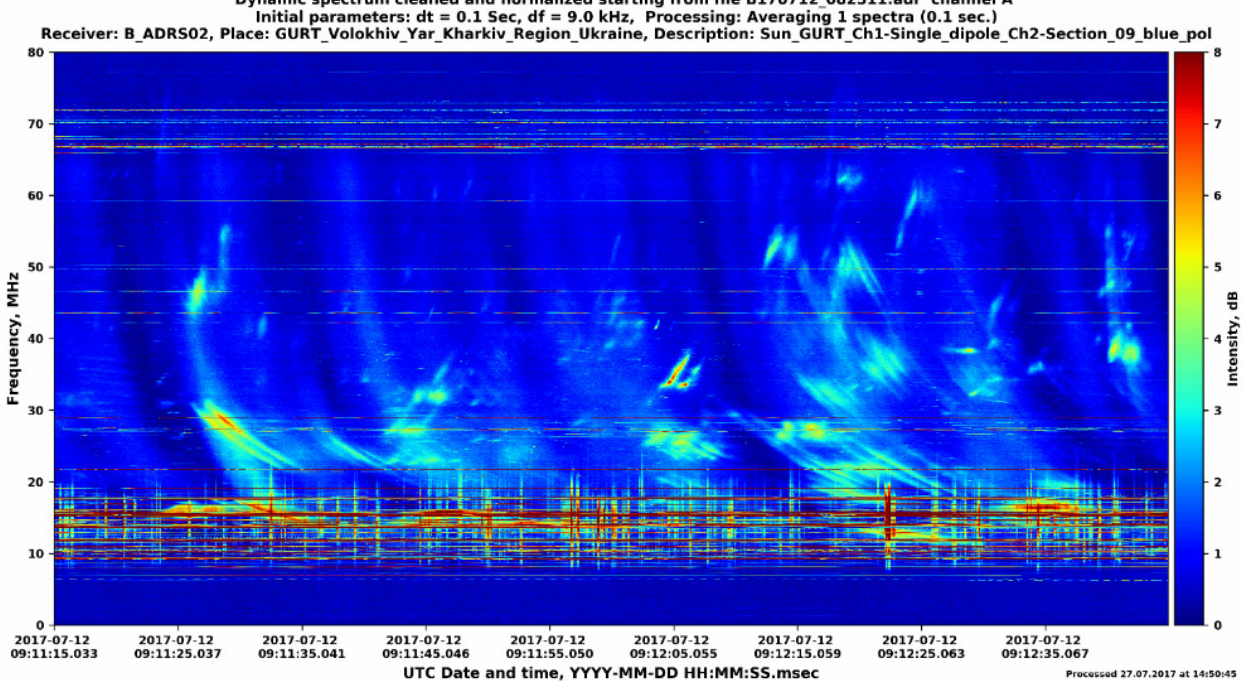

Figure 2: Examples of various bursts types in dynamic spectra recorded with the GURT active antenna.

\section{Test Measurements and Comparison with Observations by Other Instruments}

The best evidence of small-sized radio telescope performance is real observations of different type of emissions. During 2017 2018 , we have conducted several sessions of such observations when together with the GURT subarrays a single active antenna was used. The results of observations show a great variety of solar radio bursts available for studies in detail: II, III, IIIb and not only as well drifting pairs (Stanislavsky et al., 2017) are distinctly seen on dynamic spectra obtained (see records in Fig. 2). The calculated sensitivity of the GURT active antenna is quite high to prove the quality of the instrument. We have compared several spectra with ones received by the well-known Nançay Decametric Array (NDA) radio telescope. NDA has 144 passive antennas of two circular polarizations (72 antennas of right-hand and 72 of left-hand). Fig. 3 shows a type II solar burst received with a single GURT active antenna and 72 passive antennas of NDA. The quality of the data is pretty much comparable, due to the high sensitivity of antenna systems developed at IRA NASU.

\section{Possible Applications of Small Radio Telescopes}

Small-sized radio telescopes were of use at the beginning of radio astronomy but afterwards migrated to large-scale ones. Therefore, the question is natural: why do we need small-sized instruments nowadays? First of all, it should be noted that smallsized radio telescope can be a source of the immediate information on solar corona events, especially the coronal mass ejections (CME) and their velocities, affordable for many research centers, universities and colleges. Usually the data from well-known telescopes become available online within several days gap, which can be critical for some applications. Such telescopes can also serve as triggers, signalizing for dedicated advanced telescopes to switch attention in solar observations from one event to another in case of strong, diverse or interesting events. To spread all over the world, a network of small-sized radio telescopes can conduct solar observations round the clock, providing reliability of results and higher RFI stability due to observations in different RFI and ionosphere conditions. There is also a chance for arranging VLBI system of many antennas that spread over globe to perform hi-res imaging of solar corona active regions, but the perspective needs substantial hardware and network resources. At the same time, the system of solar VLBI at lower frequencies could expand capabilities of such highly-demanded instruments as LOFAR, LWA, UTR-2 and SKA. 

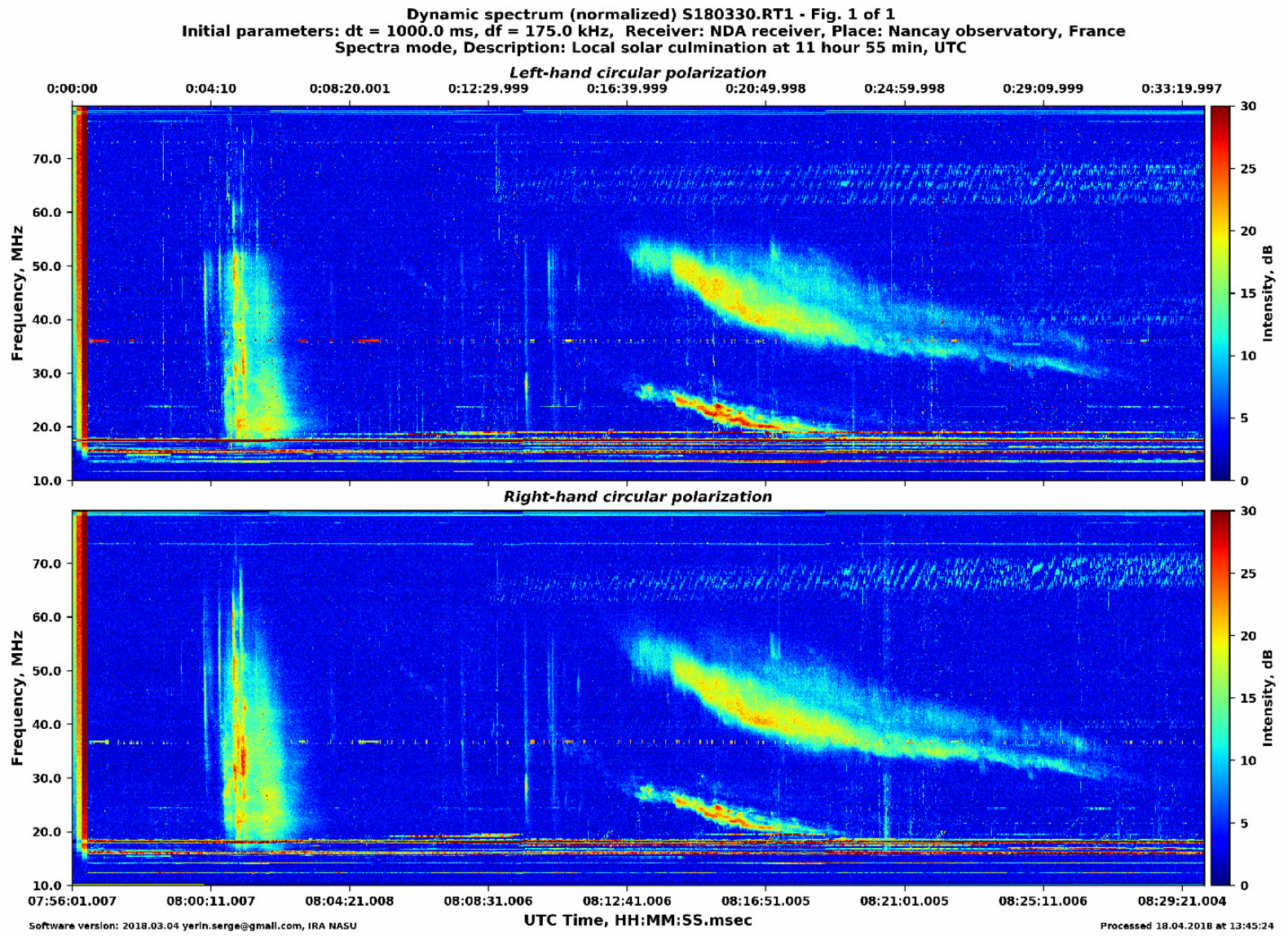

Figure 3: Dynamic spectrum of type III and II bursts obtained from the NDA observations on 30 March of 2018 simultaneously with GURT observations shown in Fig. 2.

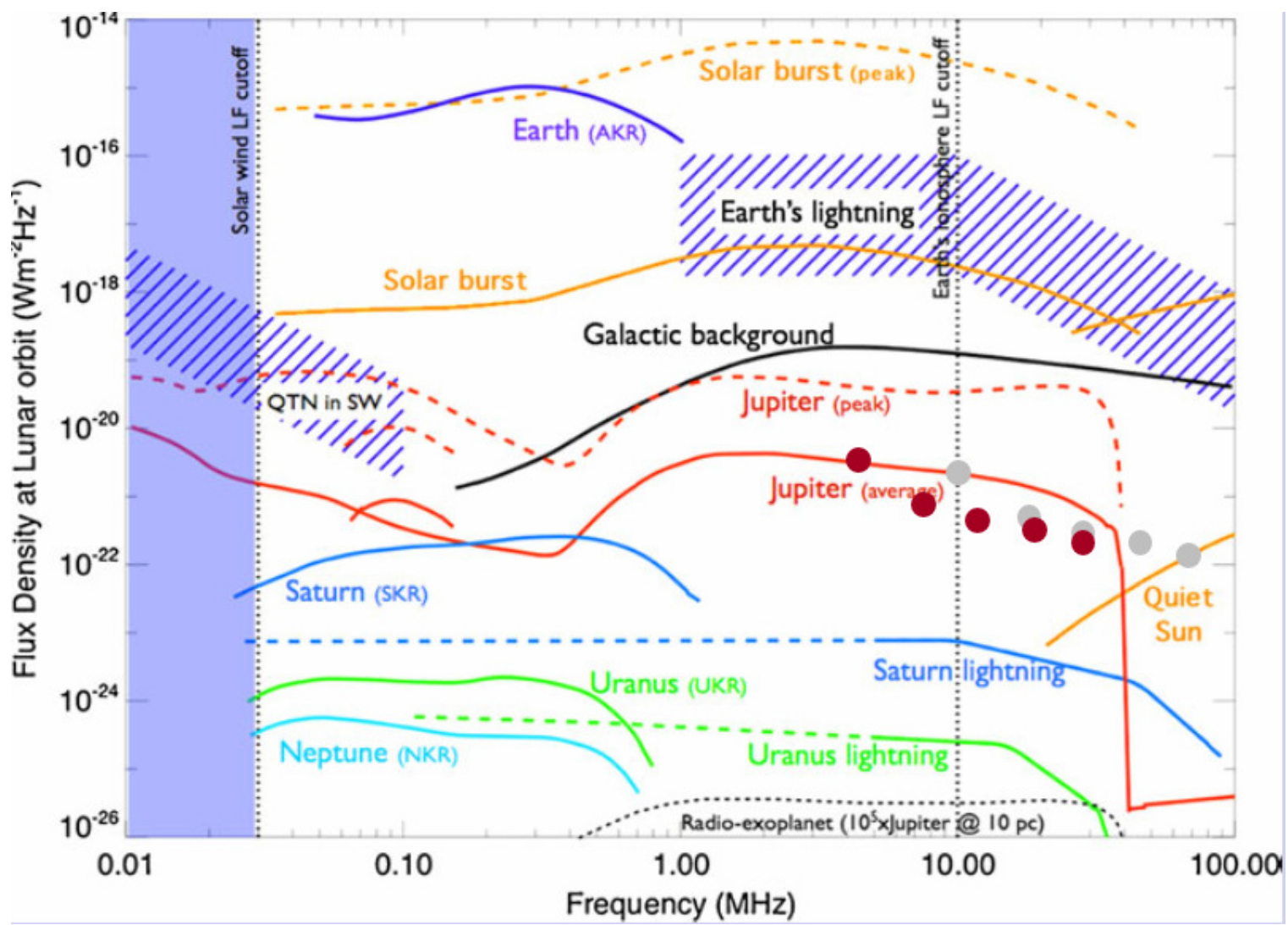

Figure 4: The levels of radio emission from different cosmic sources at the far-side of the Moon (courtesy of Mimoun et al., 2012). This figure shows estimated sensitivity of the GURT active antenna as it is (gray dots) and after frequency range shifting (red dots). 
The main advantage of sensitive wideband small-sized radio telescopes is their size, which is critical in some applications, particularly in space missions. The high cost needed to launch big and heavy instruments to space limits the possibilities of space explorations. Small sized antenna like GURT active antenna is a perfect instrument to build a space- or lunar-based radio telescope [Shkuratov et al., 2018]. The shape of the antenna allows one to build antenna arrays from them and to transport it in a folded state. As the Moon has a much weaker ionosphere, which is a main hindering factor for observations at low frequencies from the Earth, the frequency range of the active antenna can be shifted down to lower frequencies. As the higher-to-lower operational frequency ratio is a constant which limits the operational band, it is possible to shift the operational range to $4-40 \mathrm{MHz}$ range with changing the dimensions of dipole itself and matching circuits in the antenna amplifier. Such modification would allow observing solar radio emission under terrestrial ionosphere cutoff. This feature is especially useful for studies of solar drift pairs [Stanislavsky et al., 2017a and 2017b], which are known to be emitted at lower frequencies, and collect statistics of their appearance. Another advantage could be achieved by placing the radio telescope on the far side of the Moon, where manmade RFI are screened by the Moon itself [Mimoun et al., 2012].

The calculated values of GURT active antenna sensitivity were marked on the plot showing the levels of cosmic radio sources flux densities as seen from the Moon and are presented in Fig. 4. The figure clearly shows the prospect of solar emission studies with GURT active antenna on the far-side of the Moon.

\section{Conclusions}

New antenna systems in low frequency radio astronomy become more sensitive in wide frequency range, which means they can be used as small but sensitive instruments for particular observational tasks. On the example of the GURT active antenna we show the possibilities of single antennas to receive solar bursts of various types for their study at meter and decameter wavelengths. Also we discuss the possibility of using such antennas out of the terrestrial ionosphere to observe solar bursts under ionosphere cutoff. One of the most convenient places to install such a radio telescope could be the far-side of the Moon.

\section{Acknowledgments}

We would like to thank the NDA team for developing and operating the instrument and we are grateful for their open data policy. This research was partially supported by Research Grant 0118U000564 from the National Academy of Sciences of Ukraine.

\section{References}

[1] Konovalenko, A., Sodin, L., Zakharenko, V., et al.: 2016, Experim. Astron. 42, 11. doi:10.1007/s10686-016-9498-x

[2] Mimoun, D., Wieczorek, M., Alkalai, L., et al.: 2012, Experim. Astron. 33, 529-585. doi:10.1007/s10686-011-9252-3

[3] Ryabov, V., Vavriv, D., Zarka, P., Ryabov, B., Kozhin, R., Vinogradov, V., Denis, L.: 2010, Astron. Astrophys. 510, id. A16. doi: 10.1051/0004-6361/200913335

[4] Shkuratov, Y., Konovalenko, A., Zakharenko, V., et al.: 2018, Acta Astronautica, in press, doi: 10.1016/j.actaastro.2018.03.038

[5] Stanislavsky, A., Konovalenko, A., Volvach, Ya.: 2017, Res. Astron. Astrophys. 17, no. 9, 97. doi: 10.1088/16744527/17/9/97

[6] Stanislavsky, A., Volvach, Ya., Konovalenko, A., Koval, A.: 2017, Sun and Geosphere, 12, no. 2, 99-103.

[7] Tokarsky, P., Konovalenko, A., Falkovich, I., Yerin, S.: 2015, Proc. of the 45th European Microwave Conference (EuMC 2015), 7-10 September 2015. Paris, France, 1594. doi: 10.1109/EuRAD.2015.7346365

[8] Tokarsky, P., Konovalenko, A., Yerin, S.: 2017, IEEE Trans. on Antennas and Propagation. 65, no. 9, 4636-4644. doi: 10.1109/TAP.2017.2730238

[9] Zakharenko, V., Konovalenko, A., Zarka, P., et al.: 2016, J. Astron. Instrum. 05, id. 1641010. doi: $10.1142 /$ S2251171716410105 